\title{
Digitale Ordnungspolitik - Wirtschaftspolitik daten- und evidenzbasiert weiterentwickeln
}

\begin{abstract}
Die Digitalisierung bringt weitreichende Änderungen für Wirtschaft und Gesellschaft mit sich. Vor diesem Hintergrund hat das Bundesministerium für Wirtschaft und Energie als Teil der Umsetzungsstrategie „Digitalisierung gestalten“ der Bundesregierung im Dezember 2020 ein Konzeptpapier für ein ordnungspolitisches Handlungsprogramm zur Unterstützung des digitalen Wandels veröffentlicht. Dessen Kernthese ist, dass die Grundgedanken der Ordnungspolitik in der digitalen Wirtschaft gültig bleiben. Ihre Grundsätze sind bis heute Pfeiler der Sozialen Marktwirtschaft und können in einer zunehmend digitalen Wirtschaft klare Orientierung für die Wirtschaftspolitik der Zukunft bieten. Das Konzeptpapier wird vorgestellt und einer der dort skizzierten Ansätze für die Umsetzung einer digitalen Ordnungspolitik genauer beleuchtet: die daten- und evidenzbasierte Weiterentwicklung der Wirtschaftspolitik.
\end{abstract}

\begin{abstract}
Der Begriff „digitale Ordnungspolitik“ provoziert das wirtschaftspolitische Denken. Die einen halten Ordnungspolitik für ein überkommenes deutsches Konzept, was

(C) Der/die Autor:in 2021. Open Access: Dieser Artikel wird unter der Creative Commons Namensnennung 4.0 International Lizenz veröffentlicht (creativecommons.org/licenses/by/4.0/deed.de).

Open Access wird durch die ZBW - Leibniz-Informationszentrum Wirtschaft gefördert.

* An dem Artikel haben weitere Mitarbeiter:innen des BMWi mitgewirkt. Wir danken Dr. Sören Enkelmann, Dr. Sophie Gappa, Dr. Alfred Garloff, Tim Giliard, Dr. Konstantin Kolloge, Dr. Martin Weißenberger und Dr. Christian Wittneben für Beiträge und Diskussionen.
\end{abstract}

Dr. Philipp Steinberg ist Abteilungsleiter Wirtschaftspolitik im Bundesministerium für Wirtschaft und Energie, Ressortkoordinator Nachhaltigkeit sowie Leiter der Projektgruppe Wirtschaftsstabilisierungsfonds.

Dr. Nils Börnsen ist dort Referent im Referat „Grundsatzfragen der Wettbewerbspolitik, Kartellrecht, wettbewerbspolitische Fragen der Digitalisierung“ in der Abteilung Wirtschaftspolitik.

Dr. Dirk Neumann ist dort Referent im Referat "Wirtschaftspolitische Analyse" in der Abteilung Wirtschaftspolitik. sich noch nicht einmal übersetzen lässt. Andere glauben nach wie vor: Ordnungspolitik, das ist beste deutsche wirtschaftspolitische Tradition. Große Namen wie Eucken, Müller-Armack und von Hayek kommen in den Sinn. Grundpfeiler der Sozialen Marktwirtschaft sind auf diesem Gedankengerüst gebaut und haben in der Bundesrepublik als wirtschaftspolitischer Kompass gedient. Vor dem Erfahrungshintergrund gescheiterter klassischliberaler Wirtschaftsverfassungen zu Beginn des 20. Jahrhunderts war das ordoliberale Denken im Ursprung auf ein Wiederherstellen des Vertrauens in die Funktionsfähigkeit und Leistungsfähigkeit des Wettbewerbs als zentrales Element der Wirtschafts- und Gesellschaftspolitik ausgerichtet. Zugleich war es vom Bewusstsein der Defizite dieser Kernprinzipien und der Sorge vor einem Zerfall der Gesellschaft in dysfunktionale Teilordnungen geprägt. Die Ordnungspolitik erhebt den Anspruch, eine wirtschaftspolitische Gesamtordnung zu schaffen, die der Gefahr der Lähmung des Wettbewerbs durch Konzentration von zu viel Macht in entweder staatlicher oder privater Hand entgegenwirkt. Ihr Erfolgsprinzip ist, dass sie nicht blind auf die Kräfte des Marktes vertraut, sondern einen verlässlichen, wettbewerbsorientierten Ordnungsrahmen bietet, der wirtschaftliche Freiheit und sozialen Ausgleich miteinander verbindet. Walter Euckens konstituierende Prinzipien der Wirtschaftspolitik - also ein funktionsfähiges Preissystem, offene Märkte, Privateigentum, Vertragsfreiheit, Haftung und die Konstanz der Wirtschaftspolitik - ergänzt durch regulierende Prinzipien, nach denen der Staat insbesondere eine zu stark anwachsende Marktmacht oder zu große Einkommensunterschiede ausgleichen sollte, sollen ebendies gewährleisten (Eucken, 2004; sowie auch Gerken, 2000; Hasse 
et al., 2005; Pies und Leschke, 2002). Wir glauben: Damit kann - und sollte - man heute noch arbeiten. Mit dem Konzept einer zeitgemäßen Ordnungspolitik.

Wohlgemerkt, diese ordnungspolitischen Grundsätze wurden vor dem Hintergrund einer anderen wirtschaftlichen Realität erdacht als jener der heutigen Digitalisierung von Wirtschaft und Gesellschaft. Digitalisierung, das ist schneller technologischer Wandel auf globaler Ebene, der zu grundlegenden strukturellen Änderungen über Branchen- und Staatsgrenzen hinweg führt. Schlagworte wie Disruption, Plattformökonomie, Netzwerk- und Skaleneffekte, Konzentration von Marktmacht, globale SuperstarUnternehmen, Wertschöpfungsnetzwerke und digitale Ökosysteme, datenbasierte Wertschöpfung und „Überwachungskapitalismus“ (Zuboff, 2018) prägen die öffentliche Diskussion, längst auch über Fachdiskurse hinaus. Es drängt sich also die Frage auf, wie viel Orientierung die traditionelle Ordnungspolitik hier noch leisten kann.

Klar ist: Die Soziale Marktwirtschaft ist durch die digitale Transformation von Wirtschaft und Gesellschaft gefordert, neue Antworten zu liefern und den Ordnungsrahmen neu zu justieren. Man kann sich durchaus fragen, ob die Instrumente klassischer deutscher Ordnungspolitik des 20. Jahrhunderts noch im Umgang mit internationalen Tech-Giganten des 21. Jahrhunderts taugen. Oder noch grundsätzlicher: Zersetzt der digitale Wandel von Wirtschaft und Gesellschaft die Grundpfeiler der Sozialen Marktwirtschaft? Ein Beispiel: Wenn die Funktionsfähigkeit des Preismechanismus ein Kernprinzip der Wirtschaftsordnung ist, welche Konsequenz hat dann der Wandel zentraler Geschäftsmodelle der digitalen Wirtschaft zu datenbasierten und oft vermeintlich kostenfreien Leistungen, etwa bei der Nutzung sozialer Netzwerke oder Apps? Wie viel Substanz hat der insbesondere in Teilen der digitalen Wirtschaft tief verwurzelte Glaube an die Überlegenheit privatwirtschaftlicher und technologischer Lösungen für gesellschaftliche Probleme gegenüber staatlichen Ansätzen? Führt die wachsende Ubiquität von Daten zu einer Renaissance einer nun „digitalen Planwirtschaft" (Daum und Nuss, 2021), in der nicht mehr ein dezentrales Preissystem, sondern von mächtigen Plattformen gesteuerte Algorithmen Angebot und Nachfrage bestimmen und das herkömmliche Spiel der Kräfte im freien Wettbewerb ablösen?

Die Diskussion, inwieweit die Grundsätze der Ordnungspolitik auf die digitale Ökonomie übertragbar sind, wird seit einiger Zeit auch auf akademischer Ebene geführt (WalterEucken-Institut, 2020) und beschäftigt das Bundesministerium für Wirtschaft und Energie (BMWi), das - als Teil der Umsetzungsstrategie „Digitalisierung gestalten“ der Bundesregierung - im Dezember 2020 ein Konzeptpapier für ein ordnungspolitisches Handlungsprogramm zur Unterstützung des digitalen Wandels erstellt hat (BMWi, 2020a, 2021a). Die Kernthese dieses Papiers ist: Trotz allen Wandels sind die Kerngedanken der Ordnungspolitik auch in der digitalen Wirtschaft noch gültig. Ihre Grundsätze sind bis heute Pfeiler der Sozialen Marktwirtschaft und können auch in einer zunehmend digitalen Wirtschaft klare Orientierung für die Wirtschaftspolitik der Zukunft bieten.

\section{Ordnungsrahmen für die Plattformökonomie}

Ein erster Bereich, in dem sich ordnungspolitischer Handlungsbedarf bereits heute deutlich abzeichnet und der von übergeordneter Bedeutung ist, ist die zunehmende Marktmacht globaler Digitalkonzerne. Während die klassisch-liberale Schule noch vor allem auf das Problem wirtschaftlicher Machtinteressen auf staatlicher Seite ausgerichtet war und die Freiheit gegenüber dem Staat sichern wollte, ist es gerade die ordoliberale Schule, die daneben auch die Gefahr der Minderung von Wohlfahrt durch private Machtinteressen sah und das gedankliche Gerüst für die Eindämmung von Marktmacht und sozialen Ausgleich lieferte. In einer Phase der starken Konzentration von Marktmacht in digitalen Märkten ist es möglicherweise gerade diese Sensibilisierung gegenüber einer Lähmung des Wettbewerbs von privater Seite, die zentral wird. Denn die Digitalisierung scheint Plattformstrukturen als zentrales Charakteristikum vieler zukünftiger Märkte hervorzubringen, inklusive den ihr teils wohl inhärenten Monopolisierungstendenzen. Aus diesem Grund stellt die Wettbewerbspolitik - nach Eucken ein regulierendes Prinzip - auch einen wesentlichen Baustein einer digitalen Ordnungspolitik dar. Mit dem GWB-Digitalisierungsgesetz für ein fokussiertes, proaktives und digitales Wettbewerbsrecht 4.0 hat die Bundesregierung hier - aufbauend auf zahlreichen vom BMWi und von anderen in- und auch ausländischen Regierungsinstitutionen in Auftrag gegebenen Studien ${ }^{1}$ - jüngst grundlegende Schritte unternommen. Zentrale Elemente sind die Aufnahme von Daten in die Essential-Facilities-Doktrin, die Schaffung von horizontalen Datenzugangsansprüchen im Wettbewerbskontext sowie eine neue Vorschrift zur Verhinderung von missbräuchlichem, marktübergreifendem Verhalten großer Plattformunternehmen (Gatekeeper); ebenso die Fokussierung auf relevante Fälle und eine Beschleunigung der Verfahren, auch um ein oft schnelles „Kippen“ von Märkten zu verhindern. Die EU ist dabei, mit einem maßgeblich auch auf deutsche Initiative zurückgehenden

1 Für Deutschland: BMWi (2019a) und Schweitzer et al. (2018). Für die USA: Berichte des Stigler Committee on Digital Platforms (2019) und des United States Congress, House Of Representatives, Committee On The Judiciary (2020). Für Großbritannien: Digital Competition Expert Panel (2019). Für Australien: Australian Competition and Consumer Commission (2019). Für die EU: Crémer et al. (2019). 
Digital Markets Act ein speziell auf Fairness und Bestreitbarkeit von digitalen Märkten ausgerichtetes neues Instrument zu schaffen. Auch in den USA und selbst in China gewinnen wettbewerbspolitische Debatten mit Blick auf Marktmacht in digitalen Märkten deutlich an Fahrt.

Weitere zentrale Themenfelder des Konzeptpapiers, die hier nicht weiter vertieft werden, sind die Korrektur von Marktversagen - nicht zuletzt zur Steigerung von Ressourceneffizienz und des Klimaschutzes -, die Gewährleistung des sozialen Ausgleichs - insbesondere mit Blick auf den Arbeitsmarkt, die Besteuerung und den sozialen Schutz - sowie Fragen der Kontrolle, Verfügbarkeit und Sicherheit von Daten und der damit verbundenen Haftung. Gerade in Bezug auf Letzteres ist eine digitale Ordnungspolitik gefordert, einen verlässlichen Ordnungsrahmen zu gestalten. Hier wird sich neben der Europäischen Datenschutzgrundverordnung (DSGVO) ein komplementäres spezielles „Datenordnungsrecht" herausbilden, das zumindest in ausgewählten Bereichen sektorspezifisch das horizontale Wettbewerbsrecht regulatorisch ergänzt und spezifiziert (BMWi, 2019a, 34 ff.; Datenethikkommission, 2019, 79 ff., 153) - so wie sich das bereits in vielen anderen Fällen, etwa im Telekommunikationsrecht, zugetragen hat. Wesentliche Bausteine hierfür werden richtigerweise auf EU-Ebene als Teil des Digitalen Binnenmarkts gesetzt und auf nationaler Ebene flankiert. Auch die staatliche Förderung des Aufbaus einer vernetzten Dateninfrastruktur zum sicheren und vertrauenswürdigen Austausch von Daten (GAIA-X), wo dies aus dem Markt heraus nicht selbst entsteht, zählt hierzu. In allen diesen Feldern werden ordnungspolitische Prinzipien durch den digitalen Wandel zwar besonders herausgefordert, verlieren dadurch aber nicht ihre Gültigkeit. Dass sie vielmehr Orientierung bei der Gestaltung des regulatorischen Rahmens auch im Zeitalter der Digitalisierung bieten können, ist eine zentrale Aussage des Konzeptpapiers des BMWi.

\section{Evidenzbasierte Wirtschaftspolitik}

Ein weiterer, wenn auch bislang weniger im Fokus der politischen Diskussion stehender, zentraler Baustein einer digitalen Ordnungspolitik ist eine Daten- und Evidenzbasierung wirtschaftspolitischer Entscheidungen, die eine wesentliche Voraussetzung für eine effektive, effiziente und konstante Wirtschaftspolitik in der digitalen Zukunft ist.

\section{Zum Konzept}

Wenn ordnungspolitische Grundsätze insgesamt der wirtschaftspolitischen Orientierung dienen, bilden Evidenz und Datenanalyse gewissermaßen die Landkarte zum Kompass. Hier lässt sich an das anknüpfen, was die Begründer der ordoliberalen Schule in einer damals neuen Kombination von Theorie und Empirie entwickelten:
Erst das Wissen über aktuelle Entwicklungen und deren Wirkungszusammenhänge ermöglicht die Gestaltung eines kohärenten Ordnungsrahmens, die Umsetzung effektiver Maßnahmen und damit insgesamt eine Konstanz und Verlässlichkeit der Wirtschaftspolitik (Hasse et al., 2005). Das gilt erst recht bei hoher Dynamik von Transformationsprozessen in der Wirtschaft, wie sie durch den digitalen Wandel ausgelöst werden.

Dass hierbei keine banalen empirischen Fragen aufgeworfen sind, zeigt nicht zuletzt die anhaltende und changierende Debatte um das (zweite) „Produktivitätsparadoxon“2 im Kontext der Digitalisierung: Denn trotz intensiver wissenschaftlicher Diskussion bleibt es bislang dabei, dass die Erwartung spürbarer Produktivitätssteigerungen durch kostensenkende und effizienzsteigernde Prozesse infolge grundlegender Innovationen im Rahmen der digitalen Transformation mit einer ausgeprägten Produktivitätsschwäche in vielen westlichen Ländern in den vergangenen 20 Jahren kollidiert. Schwierigkeiten und Komplexitäten bei der Messung von Produktivität, unternehmerischen Transformationsprozessen und digitaler Wertschöpfung bleiben hier - neben anderen potenziellen Gründen - ein ernstzunehmender Kandidat für ein Erklärungsmuster. Mögliche Ursachen liegen dabei nicht zuletzt in der noch mangelnden Verfügbarkeit und Qualität von Daten (BMWi, 2020b).

Die Ordnungspolitik selbst kann natürlich keine Erkenntnisse für eine stärkere Daten- und Evidenzbasierung von Wirtschaftspolitik liefern. Der Einbezug von Antworten aus der empirischen Forschung steht aber in bester ordoliberaler Tradition und mitnichten im Widerspruch zum Festhalten an ordnungspolitischen Grundsätzen, wie es akademische (z.B. Fuest et al., 2006) und auch stärker öffentlich geführte Debatten der Vergangenheit in Deutschland immer wieder vermuten ließen. Auch eine Daten- und Evidenzbasierung der Wirtschaftspolitik erfolgt notwendigerweise in einem gewissen Ordnungsrahmen und bezieht sich auf diesen.

Das Konzept stärker evidenzbasierter Wirtschaftspolitik ist insofern keineswegs neu, gewinnt aber durch die Digitalisierung enorm an Bedeutung und Potenzial. Denn die Bedingungen für evidenzbasierte Entscheidungen sind heute dank stark wachsender Verfügbarkeit von Daten besser als je zuvor (Buch et al., 2019) - auch trotz neuer empirischer Herausforderungen. Big Data und neue Methoden der Datenanalyse bieten zudem bessere Instru-

2 Der Begriff etablierte sich mit der Beobachtung Robert Solows in den 1980er Jahren, dass sich (insbesondere im Dienstleistungssektor) kein positiver Zusammenhang zwischen Investitionen in die Informations- und Kommunikationstechnologie und der Produktivität auf volkswirtschaftlicher oder unternehmerischer Ebene zu zeigen schien: „You can see the computer age everywhere but in the productivity statistics" (Solow, 1987). 
mente dafür als sie der Politik jemals zuvor zur Verfügung standen. Deren Nutzung ist jedoch kein Selbstläufer. Der Blick über den Tellerrand zeigt, dass Deutschland auch hier nicht gerade zu den Vorreitern zählt. Andere Länder sind mit Modellversuchen und laufenden Projekten schon fortgeschrittener (Buch et al., 2019, 106, 109 ff.).

Die Gründe hierfür liegen, wie oft, allerdings nicht in Erkenntnisdefiziten, sondern in der Komplexität und Langwierigkeit der Erkenntnisüberführung in die breite Praxis. Letztere ist als horizontale Querschnittsaufgabe ein Mammutprojekt, das Zeit in Anspruch nimmt und verschiedenste Akteur:innen auf nahezu allen staatlichen Ebenen einbezieht. Ein Blick in den Maschinenraum des BMWi verdeutlicht aber, dass wir schon an ganz unterschiedlichen Stellen an der Verbesserung der Evidenzbasierung der Wirtschaftspolitik arbeiten.

Use cases im BMWi

- Evaluierung von Fördermaßnahmen und Regelungsvorhaben: Grundlage evidenzbasierter Wirtschaftspolitik

Es ist mittlerweile unbestritten, dass Evaluationen ein zentrales Element moderner, evidenzbasierter Wirtschaftspolitik darstellen. Durch Evaluationen soll systematisch und faktenbasiert analysiert - und für die Öffentlichkeit transparent dargelegt werden -, inwiefern die geplanten Ziele von Fördermaßnahmen, Gesetzen oder Verordnungen erreicht wurden und welche Wirkungen sie hatten. Erkenntnisse dazu sind von elementarer Bedeutung, um sicherzustellen, dass wirtschaftspolitische Ziele auf effektive und effiziente Weise erreicht werden. Evaluationen können somit auch einen Beitrag leisten, die Ausgangslage und den Bedarf für zukünftige staatliche Vorhaben besser zu identifizieren und zu konkretisieren.

Dieser Einsicht folgend hat das BMWi bereits 2011 entschieden, seine Fördermaßnahmen systematisch und regelmäßig zu evaluieren. Für die Evaluation von Gesetzen und Verordnungen hat sich die Bundesregierung 2013 zudem auf eine übergreifende Konzeption zur Evaluierung aller wesentlichen Regelungsvorhaben verständigt, die im November 2019 mit einem Beschluss des Staatssekretärsausschusses Bessere Rechtsetzung und Bürokratieabbau fortentwickelt wurde. Als erstes Bundesministerium kam das BMWi dem Auftrag des Staatssekretärsausschusses nach, Evaluationen öffentlich und zentral zugänglich zu machen. Auf der Website evaluationen-bmwi.de stellt das BMWi die Evaluationen seiner Fördermaßnahmen und Regelungsvorhaben entsprechend für jedermann zugänglich zur Verfügung. Es folgt damit auch einer Empfehlung des Wissenschaftlichen Beirats beim BMWi, die dieser, neben weiterführenden Vorschlägen, in seinem Brief an Bundesminister Altmaier im September 2019 geäußert hatte.
- Corona-Datenplattform: Evidenzbasierte Pandemiebekämpfung

Die Defizite und das Potenzial evidenzbasierter Politik zeigten sich gerade auch im Rahmen der Pandemiebekämpfung Bund und Länder haben seit dem Frühjahr 2020 zahlreiche einschneidende Maßnahmen zur Überwindung der CoronaKrise getroffen: Versammlungsverbote, Schulschließungen, Ausgangssperren und viele weitere. Anfangs wurden diese Entscheidungen auf Basis von Experteneinschätzungen und Vorgaben für Maßnahmen aus dem nationalen Pandemieplan sowie grundsätzlichen politischen Erwägungen getroffen. Im weiteren Verlauf der Pandemie entstanden jedoch zahlreiche Forschungsarbeiten und empirische Studien zur Wirksamkeit einzelner Maßnahmen. Dabei beruhten die meisten Arbeiten allerdings auf Daten und Auswertungen aus dem Ausland, da in Deutschland lange Zeit die einzelnen Eindämmungsmaßnahmen auf Bundes-, Landes- und Kreisebene nicht systematisch erfasst wurden und Daten zum Infektionsgeschehen, zur Auslastung der Intensivbetten oder zu Todesfällen nur umständlich und über mehrere Quellen verteilt zugänglich waren. Mit der Corona-Forschungsdatenplattform (corona-datenplattform.de) hat das BMWi ein umfangreiches und systematisches Datenangebot geschaffen, das es der Wissenschaft erlaubt, die Wirkung einzelner Maßnahmen empirisch und systematisch zu überprüfen. Aktuell wird die Datenbank von mehr als 300 Wissenschaftler:innen aus unterschiedlichen Forschungszweigen genutzt und in Forschungsarbeiten, wie z. B. des ifo Instituts, verwendet. So wird die Grundlage für eine evidenzbasierte Wirtschafts- und Krisenpolitik gelegt. Wegen des hohen Interesses an den Daten und aus Gründen der Transparenz wird das Datenangebot - ganz im Sinne der Open-Data-Strategie der Bundesregierung - seit Juli 2021 über das Statistische Bundesamt öffentlich zur Verfügung gestellt.

\section{- Reallabore: Innovationen ermöglichen, Regulierung weiterentwickeln}

Aufgrund der rasanten Geschwindigkeit des digitalen Wandels ist es für die Gesetzgebung heute herausfordernder denn je, mit den aktuellen Entwicklungen Schritt zu halten. Deshalb brauchen wir auch in der Gesetzgebung neue Freiräume zum Experimentieren und Lernen, um das Wissen über Wirkzusammenhänge von Innovationen und Regulierung sukzessive weiter zu verbessern und um Regulierung agil weiterzuentwickeln. Reallabore (Regulatory Sandboxes) machen es möglich, neue Technologien oder Geschäftsmodelle unter realen Bedingungen zu erproben, die der geltende Rechtsrahmen regulatorisch noch nicht berücksichtigt oder sogar hemmt. Experimentierklauseln schaffen dafür die notwendigen rechtlichen Freiräume. Mit ihren Ergebnissen tragen Reallabore dazu bei, den Rechtsrahmen evidenzbasiert weiterzuentwickeln. So dienen Reallabore als 
Impulsgeber für Innovationen und bilden ein weiteres wichtiges Instrument einer digitalen Ordnungspolitik.

Im Rahmen der BMWi-Reallabore-Strategie arbeitet das BMWi eng mit den betroffenen Ministerien zusammen, um bessere rechtliche Rahmenbedingungen für Reallabore zu schaffen. Hierzu hat die Bundesregierung am 13. April 2021 im Paket für Bürokratieerleichterungen beschlossen, in Zukunft für jedes Gesetz zu prüfen, ob durch die Aufnahme von Experimentierklauseln neue Reallabore ermöglicht werden können - wie etwa zuletzt für die Erprobung von autonomem Fahren oder digitalen Identifizierungsverfahren beim Hotel-Check-in. Auch auf europäischer Ebene müssen wir Möglichkeiten für Reallabore schaffen, etwa für Blockchain und Künstliche Intelligenz. Hierzu haben die 27 EUMitgliedstaaten die EU-Kommission mit ihren Ratsschlussfolgerungen zu Reallaboren und Experimentierklauseln am 16. November 2020 aufgefordert. Ebenso unterstützt das BMWi die Reallabore-Praxis durch Vernetzung und Veranstaltungen (Netzwerk Reallabore), durch aktuelle Informationen und Leitfäden (Handbuch Reallabore, Praxishilfe zum Datenschutz) sowie durch den regelmäßig stattfindenden "Innovationspreis Reallabore“, der herausragende Reallabore würdigt und sichtbar macht (BMWi, 2021b).

- Basisregister, bundeseinheitliche Wirtschaftsnummer: Qualitätssteigerung der Registerdaten

Evidenzbasierte Wirtschaftspolitik funktioniert nicht ohne qualitativ hochwertige Daten. Dies gilt auch für Unternehmensdaten, angefangen bei den Datenbeständen in amtlichen Registern. Aktuell gibt es in Deutschland rund 120 Register mit Unternehmensbezug, ein Austausch von Informationen zwischen den Registern erfolgt üblicherweise jedoch nicht. Ein Grund hierfür ist, dass bisher keine registerübergreifende Identifikationsnummer existiert. Mit dem im Juni 2021 von der Gesetzgebung verabschiedeten Unternehmensbasisdatenregistergesetz wird die Grundlage für eine moderne und vernetzte Registerlandschaft geschaffen. Das Gesetz sieht vor, ein zentrales Basisregister für Unternehmensstammdaten beim Statistischen Bundesamt zu errichten und zu betreiben. Zudem wird die WirtschaftsIdentifikationsnummer als bundeseinheitliche Wirtschaftsnummer in Deutschland eingeführt. Das Vorhaben sorgt für Entlastungen bei Unternehmen, für Effizienzsteigerungen in der Verwaltung und für Qualitätsverbesserungen in der Statistik. Damit schaffen wir ein breites Fundament für bessere Datennutzung, -verfügbarkeit und -verknüpfung für Unternehmen, Forschung und Verwaltung.

\section{- Projektion: Frühindikatoren, Nowcasting, Big Data}

Evidenzbasierte Wirtschaftspolitik benötigt zudem zeitnahe und präzise Informationen über die wirtschaftliche
Entwicklung, um ihre Handlungen auf den jeweiligen Zustand der Volkswirtschaft möglichst zielgenau abstimmen zu können. Idealerweise könnten Entscheidungstragende sogar auf tagesaktuelle Echtzeitdaten zurückgreifen. Tatsächlich werden viele relevante Daten allerdings erst mit Verzögerung veröffentlicht (BMWi, 2019b). So stehen erste amtliche Daten zum Bruttoinlandsprodukt (BIP) erst 30 Tage nach Ablauf des jeweiligen Quartals zur Verfügung. In der Konjunkturanalyse und -prognose werden daher in der Regel eine Reihe höherfrequenter Konjunkturindikatoren ausgewertet, die früher vorliegen als die Quartalsdaten und eine Einschätzung der aktuellen wirtschaftlichen Entwicklung ermöglichen. Darunter sind sowohl monatliche Statistiken beispielsweise zur Produktion oder der Auftragslage als auch umfragebasierte Stimmungsindikatoren. Weitere Hilfsmittel zur zeitnahen Auswertung sind ökonometrische Nowcasting-Modelle oder Flash-Schätzungen, die aktuelle Indikatoren automatisch auswerten und daraus eine rein technische Prognose der wirtschaftlichen Entwicklung im laufenden Quartal berechnen können. Am BMWi erweitert ein solches Modell seit einiger Zeit das bestehende Analyseinstrumentarium; die Ergebnisse werden regelmäßig in den „Schlaglichtern der Wirtschaftspolitik" des BMWi veröffentlicht und kommentiert.

In den vergangenen Jahren haben Fortschritte im Bereich der künstlichen Intelligenz zusammen mit der Digitalisierung fast aller Lebensbereiche zu neuen Chancen für die makroökonomische Analyse geführt. So können mit speziellen statistischen Methoden aus großen, zunächst häufig unstrukturierten Datenquellen (Big Data) wertvolle neue Informationen gewonnen werden. Das BMWi lotet derzeit anhand eines Forschungsprojekts das Potenzial solcher Methoden für die Beobachtung, Analyse und Projektion der konjunkturellen Entwicklung in Deutschland aus. Mit dem Projekt soll auch der deutschen Konjunkturforschung ein Impuls gegeben werden, sich weiterhin intensiv mit den Themen Digitalisierung und Big Data zu beschäftigen. Allerdings können solche stark datengetriebenen Analysen Expertenwissen nicht ersetzen. So können qualitative Sonderfaktoren und Politikmaßnahmen im Modell nur schwer erfasst werden. Während der Corona-Krise zeigte sich dies beispielsweise bei der Anordnung notwendiger Eindämmungsmaßnahmen, die ex ante in Modellen nur schwer abzubilden sind. Umso größere Bedeutung kommt der Interpretation der Daten und Ergebnisse zu.

\section{- Foresight: Szenarienentwicklung für eine langfristig vo-} rausschauende Wirtschaftspolitik

Zur Evidenzbasierung zählt auch, langfristige Planungen auf fundiertere Grundlagen zu stellen. Um mögliche längerfristige wirtschaftliche Auswirkungen der Digitalisierung zu analysieren, hat das BMWi 2019 einen Strategischen Voraus- 
schauprozess initiiert. Die „Strategische Vorausschau“ (Strategic Foresight) ist ein Feld der Zukunftsforschung, das sich mit zukünftigen gesellschaftlichen, politischen oder technologischen Entwicklungen beschäftigt. Dabei können auch Szenarien betrachtet werden, die zunächst noch von Entwicklungen in der Anfangsphase ausgehen oder unwahrscheinlich erscheinen, aber dennoch unter gewissen Entwicklungspfaden vorstellbar sind. Ziel des ForesightAnsatzes ist dabei nicht, ein bestimmtes Ergebnis möglichst genau vorherzusagen; vielmehr sollen mehrere mögliche „Zukünfte“ beleuchtet werden. Strategische Vorausschau dient somit als Instrument für die strategische Planung, um auf mehrere mögliche Entwicklungen vorbereitet zu sein.

Ziel des Prozesses im BMWi ist es, Zukunftsszenarien zu den wirtschaftlichen Perspektiven der Digitalisierung für einen Zeitraum von zehn bis 15 Jahren zu entwickeln und daraus Handlungsempfehlungen für die Anpassung des Ordnungsrahmens der Sozialen Marktwirtschaft in der digitalen Zukunft abzuleiten. Dazu wurden zunächst verschiedene Schlüsseltechnologien identifiziert, deren weitere Entwicklungen maßgeblichen Einfluss auf die Entwicklungsrichtung der Digitalisierung in Deutschland haben könnten, unter anderem Autonome Systeme, Big Data, Internet der Dinge, Künstliche Intelligenz. Letztere wurden unter Berücksichtigung übergeordneter Einflussfaktoren (aus Wirtschaft, Gesellschaft, Politik, Recht, Umwelt etc.) herausgearbeitet, an die sich eine Wechselwirkungsanalyse der Technologien anschloss. Davon ausgehend wurden sechs konsistente Szenarien identifiziert, die aus wirtschaftspolitischer Sicht besonders relevant erscheinen. Sie zentrieren sich um wesentliche Entwicklungsrichtungen in den Bereichen Plattformen, Industrie 4.0, Systemwettbewerb und Technologieführerschaft, digitalisierter Alltag sowie Nachhaltigkeit. Die Ergebnisse wurden auf einer öffentlichen Abschlusskonferenz im Juni präsentiert und werden in die weitere Arbeit des BMWi (2021c) einfließen.

\section{Evidenzbasierte Wirtschaftspolitik - quo vadis?}

So viel auch schon angestoßen und in Arbeit ist, klar ist auch: Zur Etablierung einer umfassenden und evidenzbasierten digitalen Ordnungspolitik bleibt noch viel zu tun. Einige weitere konkrete Aufgabenfelder drängen sich aus wirtschaftspolitischer Sicht auf:

\section{Wirtschaftsstatistik-Agenda}

Um die Datenlage für Wissenschaft und Forschung zu verbessern, wäre eine spezielle Wirtschaftsstatistikagenda für Wirtschafts- und Sozialdaten hilfreich. In enger Abstimmung mit Datenproduzierenden, Forschungsdatenzentren (FDZ), Wissenschaft und Politik könnten Defizite benannt, Lösungsvorschläge generiert und gebündelt werden. In der
Datenökonomie des 21. Jahrhunderts könnten so erforderliche Maßnahmen aufgezeigt und konkrete Umsetzungsschritte definiert werden, um eine umfassende Evidenzbasierung der Wirtschaftspolitik langfristig zu unterstützen.

Durch eine solche Agenda sollten substanzielle Fortschritte beim Datenzugang und der Nutzung von Wirtschaftsdaten erzielt werden. Daten mit Relevanz für die wirtschaftspolitische Beratung sollten zukünftig einfacher, häufiger, rechtssicher und in höherer Qualität für Forschungszwecke eingesetzt werden. Ein Zugang zu und die Verknüpfung von Mikrodaten sollte systematischer als bisher ermöglicht und neue Datenquellen und Erhebungswege sollten kontinuierlich erschlossen werden. Dies ist mit der politischen Forderung nach einer Erweiterung des Forschungsprivilegs bei der Datennutzung verknüpft. Die Agenda sollte zudem das Bewusstsein im politischen Raum für die Bedeutung einer leistungsfähigen wissenschaftlichen Wirtschaftsdateninfrastruktur für Deutschland stärken. Dabei wäre die Wirtschaftsstatistikagenda ein Teil der Konkretisierung und Umsetzung der Datenstrategie der Bundesregierung und eine Ergänzung des Aktionsplans Forschungsdaten des BMBF.

\section{Reallabore-Gesetz}

Trotz der wachsenden Bedeutung von Reallaboren und neuer verbesserter rechtlicher Möglichkeiten in verschiedenen Innovationsbereichen fehlt es bislang an einheitlichen Standards für Reallabore. Anforderungen und Zugangsbedingungen für Unternehmen und andere Innovatoren unterscheiden sich oft erheblich; Rechtslage und Genehmigungspraxis sind gerade für kleine und mittlere Unternehmen sowie Start-ups oft unübersichtlich. Vor diesem Hintergrund entwickeln wir derzeit einen Vorschlag für ein mögliches übergreifendes ReallaboreGesetz. Ziel des Vorschlags ist, durch Vereinheitlichung, Präzisierung und Verbesserung der rechtlichen und prozessualen Grundlagen für mehr regulatorische Agilität zu sorgen und die Anwendung und den Nutzen von Reallaboren für alle Beteiligten nachhaltig zu stärken.

\section{Chief Data Scientists in den Bundesministerien}

Die Datenstrategie der Bundesregierung, in die sich die evidenzbasierte digitale Ordnungspolitik eingliedert, sieht zu Recht die Etablierung von Chief Data Scientists und experimentellen Datenlaboren in den Bundesministerien vor. Sie sollen für eine bessere Erschließung und Nutzung von Datenquellen sorgen und als Schnittstellen zwischen technischem und fachlichem Know-how dienen. Richtig etabliert können solche Schnittstellen zum Katalysator für eine bessere Datennutzung und Evidenzbasierung auch in der Wirtschaftspolitik werden. 


\section{Digitale Ordnungspolitik auf EU-Ebene}

Nicht weiter vertieft werden können hier die zahlreichen Bezüge zur europäischen Ebene, die für die digitale Wirtschaft ohne Zweifel von zentraler Bedeutung ist. Der Vollständigkeit halber erwähnt sei aber, dass die EU mit den drei geplanten Rechtsakten Digital Markets Act, Digital Services Act und Data Governance Act dabei ist, zentrale Elemente eines europäischen Ordnungsrahmens für die digitale Wirtschaft neu zu gestalten. Wesentlicher Baustein des Data Governance Act wird auch eine stärkere Harmonisierung der Weiterverwendungsmöglichkeiten für geschützte Daten öffentlicher Stellen durch Strukturen wie die - in Deutschland schon bekannten - Forschungsdatenzentren sein; nicht zuletzt auch, um den Zugang zu Daten für die Forschung europaweit weiter zu verbessern. Daneben wird es im weiterhin geplanten Data Act der EU unter anderem darum gehen, den Zugang zu Daten für die Forschung zu erleichtern. Zudem ist im Rat der Europäischen Union die stärkere Verwendung von neuen Datenanalyse-Tools im Rahmen der Grundsätze für bessere Rechtsetzung auf EU-Ebene (Better Regulation) in Diskussion. Auch hier wird die Entwicklung zu einer stärker evidenzbasierten Politik vorangetrieben.

\section{Literatur}

Australian Competition and Consumer Commission (2019), Digital platforms inquiry - final report, https://www.accc.gov.au/publications/ digital-platforms-inquiry-final-report (23. Juni 2021).

BMWi (2019a), Ein neuer Wettbewerbsrahmen für die Digitalwirtschaft Bericht der Kommission Wettbewerbsrecht 4.0, https://www.bmwi. de/Redaktion/DE/Publikationen/Wirtschaft/bericht-der-kommission-wettbewerbsrecht-4-0.html (23. Juni 2021).

BMWi (2019b), Nowcasting: Ein Echtzeit-Indikator für die Konjunkturanalyse, Schlaglichter der Wirtschaftspolitik, 7.

BMWi (2020a), Digitale Ordnungspolitik - Konzept für ein ordnungspolitisches Handlungsprogramm des BMWi zur Unterstützung des digitalen Wandels, https://www.bmwi.de/Redaktion/DE/Downloads/J-L/ konzeptpapier-digitale-ordnung.html (23. Juni 2021).

BMWi (2020b), Produktivität: Kommt nun der große Schub?, Schlaglichter der Wirtschaftspolitik, 10.

BMWi (2021a), Ordnungsrahmen für die digitalisierte Wirtschaft, Schlaglichter der Wirtschaftspolitik, 1.

BMWi (2021b), Reallabore - Testräume für Innovation und Regulierung, https://www.bmwi.de/Redaktion/DE/Dossier/reallabore-testraeumefuer-innovation-und-regulierung.html (23. Juni 2021).
BMWi (2021c), Foresight-Abschlusskonferenz, https://www.bmwi.de/ Redaktion/DE/Veranstaltungen/2021/foresight-abschlusskonferenz. html (23. Juni 2021).

Buch, C. M., K. Patzwaldt, R. T. Riphahn und E. Vogel (2019), Verstehen - Entwickeln - Testen - Verbessern: Rahmenbedingungen für evidenzbasierte Politik, Wirtschaftsdienst, 99(2), 106-112, https://www. wirtschaftsdienst.eu/inhalt/jahr/2019/heft/2/beitrag/verstehen-entwickeln-testen-verbessern-rahmenbedingungen-fuer-evidenzbasierte-politik.html (7. Juli 2021).

Crémer, J., Y.-A. de Montjoye und H. Schweitzer (2019), Competition for the Digital Era, Studie im Auftrag der EU-Kommission, https://ec.europa.eu/ competition/publications/reports/kd0419345enn.pdf (23. Juni 2021).

Datenethikkommission (2019), Gutachten der Datenethikkommission, https://www.bmi.bund.de/SharedDocs/downloads/DE/publikationen/themen/it-digitalpolitik/gutachten-datenethikkommission.pdf (23. Juni 2021).

Daum, T. und S. Nuss (Hrsg.) (2021), Die unsichtbare Hand des Plans Koordination und Kalkül im digitalen Kapitalismus, Dietz.

Digital Competition Expert Panel (2019), Unlocking digital competition, Report of the Digital Competition Expert Panel, https://www.gov.uk/ government/publications/unlocking-digital-competition-report-ofthe-digital-competition-expert-panel (23. Juni 2021).

Eucken, W. (2004), Grundsätze der Wirtschaftspolitik, UTB, 7. Aufl.

Fuest, C., N. Goldschmidt, B. Lucke, B. P. Priddat und G. G. Wagner (2006), Abkehr von der Ordnungspolitik in der Ökonomie?, Wirtschaftsdienst, 86(1), 7-25, https://www.wirtschaftsdienst.eu/inhalt/ jahr/2006/heft/1/beitrag/abkehr-von-der-ordnungspolitik-in-deroekonomie.html (7. Juli 2021).

Gerken, L. (Hrsg.) (2000), Walter Eucken und sein Werk: Rückblick auf den Vordenker der sozialen Marktwirtschaft, Mohr Siebeck, 1. Aufl.

Hasse, R. H., H. Schneider und K. Weigelt (Hrsg.) (2005), Soziale Marktwirtschaft: Ökonomische Grundlagen und Funktionsweise, Lexikon Soziale Marktwirtschaft, Wirtschaftspolitik von A bis Z, UTB, 2. Aufl.

Pies, I. und M. Leschke (Hrsg.) (2002), Walter Euckens Ordnungspolitik, Mohr Siebeck, 1. Aufl.

Schweitzer, H., J. Haucap, W. Kerber und R. Welker (2018), Modernisierung der Missbrauchsaufsicht für marktmächtige Unternehmen, ProjektEndbericht im Auftrag des BMWi, https://www.bmwi.de/Redaktion/ DE/Publikationen/Wirtschaft/modernisierung-der-missbrauchsaufsicht-fuer-marktmaechtige-unternehmen.pdf (23. Juni 2021).

Solow, R. (1987), We'd better watch out, New York Times Book Review, 12. Juli, 36 .

Stigler Committee on Digital Platforms (2019), Final Report, https://www. chicagobooth.edu/research/stigler/news-and-media/committee-ondigital-platforms-final-report (23. Juni 2021).

United States Congress, House Of Representatives, Committee On The Judiciary (2020), Investigation Of Competition In Digital Markets, https://judiciary.house.gov/uploadedfiles/competition_in_digital markets.pdf (23. Juni 2021).

Walter-Eucken-Institut (2020), Digitale Ordnungspolitik, https://www. eucken.de/veranstaltungen/digitale-ordnungspolitik/ (23. Juni 2021).

Zuboff, S. (2018), Das Zeitalter des Überwachungskapitalismus, Campus.

Title: Digital Governance - Further Developing Economic Policy Based on Data and Evidence

Abstract: Digitalisation is bringing about far-reaching changes for the economy and society. Against this backdrop, the Federal Ministry for Economic Affairs and Energy published a concept paper for a regulatory action programme to support the digital transformation in December 2020 as part of the Federal Government's implementation strategy "Shaping Digitalisation". Its core thesis is that the basic ideas of regulatory policy remain valid in the digital economy. Its principles are still pillars of the social market economy today and can provide clear orientation for economic policy in the future in an increasingly digital economy. One of the paper's approaches to the implementation of a digital regulatory policy is examined in more detail: the data- and evidence-based further development of economic policy. JEL Classification: B25, 033, 038 